Published in final edited form as:

Arthritis Rheum. 2013 July ; 65(7): 1843-1852. doi:10.1002/art.37977.

\title{
Glucosamine Activates Autophagy In Vitro and In Vivo
}

\author{
Beatriz Caramés $^{\mathrm{a}, \mathrm{b}}$, William B. Kiosses ${ }^{\mathrm{c}}$, Yukio Akasaki ${ }^{\mathrm{a}}$, Diana C. Brinson ${ }^{\mathrm{a}}$, William Eap ${ }^{\mathrm{a}}$, \\ James Koziol ${ }^{\mathrm{a}}$, and Martin K. Lotz ${ }^{\mathrm{a}}{ }^{*}$ \\ aDepartment of Molecular and Experimental Medicine, The Scripps Research Institute, La Jolla, \\ $\mathrm{CA}$ \\ bOsteoarticular and Aging Research Laboratory, INIBIC-Complejo Hospitalario Universitario A \\ Coruña, Spain \\ ${ }^{\circ}$ Core Microscopy Facility, The Scripps Research Institute, La Jolla, CA, USA
}

\begin{abstract}
Objectives-Aging-associated changes in articular cartilage represent a main Osteoarthritis (OA) risk factor. Autophagy is an essential cellular homeostasis mechanism. Aging-associated or experimental defects in autophagy contribute to organismal and tissue specific aging while enhancement of autophagy may protect against certain aging related pathologies such as OA. The objective of this study was to determine whether glucosamine (GlcN) could activate autophagy.
\end{abstract}

Methods-Chondrocytes from normal human articular cartilage were treated with GlcN (0.1-10 $\mathrm{mM}$ ). Autophagy activation and phosphorylation levels of Akt, FoxO3 and ribosomal protein S6 (prbS6) were determined by Western blotting. Autophagosome formation was analyzed by microscopy. Transgenic reporter mice with green fluorescent protein fused to LC3 (GFP-LC3 mice) were used to test changes in autophagy in response to starvation and GlcN administration.

Results-GlcN treatment of chondrocytes activated autophagy as indicated by increased of LC3II levels, formation of LC3 puncta and increased LC3 turnover. This was associated with GlcNmediated inhibition of Akt, FoxO3 and mTOR pathway. Administration of GlcN to GFP-LC3 mice markedly activated autophagy in articular cartilage.

Conclusions-GlcN modulates molecular targets of the autophagy pathway in vitro and in vivo and the enhancement of autophagy was mainly dependent on the Akt/FoxO and mTOR pathway. These findings suggest that GlcN is an effective autophagy activator and motivate future studies on its efficacy in modifying aging-related cellular changes and supporting joint health.

\section{Keywords}

Osteoarthritis; cartilage; autophagy; glucosamine

\footnotetext{
*Correspondence to Department of Molecular and Experimental Medicine, MEM-161, The Scripps Research Institute, 10550 North Torrey Pines Road, La Jolla, CA 92037, USA; mlotz@scripps.edu.

Competing interests None.

Ethics approval This study was conducted with the approval of the Human Subjects Committee and the Institutional Animal Care and Use Committee at The Scripps Research Institute.

Author contributions All authors were involved in drafting the manuscript and all authors approved the final version. Dr. Lotz had full access to all data and takes responsibility for the integrity of the data and the accuracy of the data analysis. Study conception and design. Lotz, Carames Acquisition of data. Carames, Brinson, Kiosses, Akasaki, Eap Analysis and interpretation of data. Koziol, Carames, Kiosses, Lotz
} 


\section{INTRODUCTION}

Joint aging represents a major risk factor for the development of osteoarthritis, the most prevalent joint disease $(1,2)$. Currently, disease-modifying therapies are not available. Pharmacological management is limited to anti-inflammatory and pain medications (3). Dietary supplements are also widely used (4) although their efficacy as symptom or disease modifying therapies in patients with established or advanced disease continues to be debated (5).

There have been important recent advances in the understanding of pathogenesis mechanisms that are activated in established OA and lead to cartilage degradation and these include cell death, abnormal chondrocyte activation and differentiation $(6,7)$. Signaling mechanisms and molecules that regulate these pathways provide a large number of therapeutic targets that were validated in preclinical models and tested in clinical trials $(8$, 9 ). These OA clinical trials on disease modification failed to provide conclusive evidence for efficacy $(8,9)$. Potential explanations for these outcomes are that the therapeutic targets were highly specific or represent only a minor component in the pathogenesis network and thus do not provide sufficient impact on the overall disease process. It is also possible that the advanced disease of many patients that were enrolled in these trials is not sensitive to pharmacological modification or that it requires combination with simultaneous correction of abnormal biomechanical loading.

This has led to a shift in emphasis on understanding earlier events in the disease process and mechanisms of joint homeostasis or joint health (10). Conceptually, cartilage homeostasis depends on a normal composition of its extracellular matrix (ECM) to allow normal biomechanical function. Normal ECM composition depends on the presence of adequate numbers of cells, which produce appropriate amounts of specific ECM components. Cells in articular cartilage and other postmitotic tissues with a very low rate of proliferation depend on homeostasis mechanisms to ascertain cell survival and normal biosynthetic function.

Autophagy is an essential cellular homeostasis mechanism for the removal of dysfunctional cellular organelles and macromolecules (11). The autophagy pathway is integrated with multiple signal transduction pathways that respond to nutrient supply, energy balance, cytokines and growth factors (11). The key switch for activating the autophagy cascade is the serine threonine kinase mammalian target of rapamycin (mTOR) in mTOR complex 1 (mTORC1) (12). Inhibition of mTORC1 leads to autophagy initiation. The first step is formation of the phagophore, a double membrane structure, which is driven by the Beclin1 associated class III PI3 kinase with phosphatidylinositol3-phosphate-containing vesicles. Substrate recognition occurs during this process. The phagophore undergoes elongation and completion driven by two ubiquitin-related conjugation systems, the LC3-

phosphatidylethanolamine conjugation of LC3, resulting in the formation of the autophagy marker LC3-II, and the Atg12 and Atg5 conjugation. The autophagosome that is enclosed by double or multilayered membranes then fuses with the lysosome. The enclosed cargo is degraded and the constituents are released and utilized for biosynthesis or as sources of energy (11). mTORC1 also regulates protein synthesis by phosphorylating eukaryotic initiation factor 4E-binding protein 1 (4E-BP1) and ribosomal S6 protein kinase (S6K1, also known as p70S6K), which phosphorylates ribosomal protein s6 (13).

Defects in autophagy lead to tissue degeneration similar to those associated with aging while autophagy activation can prolong lifespan and reduce disease severity in model organisms (14). Joint aging in humans and mice is associated with reduced expression of the autophagy regulators Ulk1, Beclin1 and LC3 (15). Excessive mechanical load also compromises autophagy (16). The mTORC1 inhibitor and autophagy activator rapamycin enhances 
autophagy in cartilage and prevents against tissue destruction in vitro (16) and in experimental OA in mice (17). These observations support the general concept that autophagy activation may support joint health and protect against the development of OA (10).

Drugs such as rapamycin are useful tools for studies in OA models but potential adverse events (18) pose a challenge for chronic use to prevent or treat OA. Glucosamine is safe during long-term administration in humans $(19,20)$, including subjects with impaired glucose tolerance or diabetes (21). It is effective in animal models of OA (22-25) and has anti-inflammatory and anabolic effects on cartilage cells (26-28).

The objective of this study was to determine whether glucosamine modulates autophagy in cartilage cells in vitro and in vivo.

\section{MATERIALS AND METHODS}

\section{Materials}

The following antibodies were used for Western blotting: LC3A/B, phospho-Akt and phospho-S6 ribosomal from Cell Signaling Technology (Danvers, MA), phospho-FoxO3 from Abcam (Cambridge, MA) and Glyceraldehyde-3-phosphate dehydrogenase (GAPDH) from Invitrogen (Grand Island, NY). For immunocytochemistry (ICC) studies: anti-LC3 from MBL International (Woburn, MA) and Rabbit IgG Alexa fluor-488 from Invitrogen. For autophagy flux studies: Bafilomycin A1 was purchased from LC Laboratories (Woburn, MA), Chloroquine from Sigma (St. Louis, MO). Glucosamine-HCl was provided by Cargill (Eddyville, IA).

\section{Chondrocyte isolation and culture}

Human tissues were procured under approval by the Scripps Human Subjects Committee. At autopsy, articular cartilage was collected from the femoral condyles and the tibial plateaus from donors $($ age $<50)$ without history of joint disease and macroscopically normal cartilage surfaces. Chondrocytes were isolated and cultured as described previously (16). Firstpassage chondrocytes were used in the experiments.

Cells were plated in 6 well plates $0.25 \times 10^{6}$ in DMEM containing $10 \%$ calf serum and incubated overnight before autophagy modulators were added in DMEM $2 \%$ calf serum and incubated for indicated times before harvesting. Monolayer cell cultures were washed once with room temperature HBSS before lysis in the plates with 6M Urea / 2\% SDS. Cell lysates were sonicated at $4^{\circ} \mathrm{C}$ in a Sonic Dismembrator Model 100 (Fischer Scientific) by using program 2 with three consecutive cycles, each for 3-5 seconds. Protein concentrations were determined with the Pierce BCA Protein Assay kit (Thermo Scientific, Rockford IL).

\section{LC3 turnover assay}

The estimation of autophagic flux is important to distinguish increased autophagosome formation from impaired degradation. To measure autophagic flux, cells were incubated with GlcN in the absence or presence of vacuolar $\mathrm{H}^{+}$-ATPase inhibitor Bafilomycin A1 or Chloroquine to prevent lysosomal acidification 4 , followed by western blot analysis using LC3 antibody.

\section{Immunocytochemistry}

Cells were treated with $10 \mathrm{mM} \mathrm{GlcN}$ for 8 and 24 hours, fixed with $4 \%$ paraformaldehyde/ PBS for $10 \mathrm{~min}$ at room temperature (RT), permeabilized with $0.5 \%$ Triton X-100 in PBS at RT for 15 min and incubated with anti-LC3 (1:500, MBL International) at RT for $1 \mathrm{~h}$. A 
fluorescence dye-conjugated secondary antibody (Rabbit IgG-Alexa fluor 488, Invitrogen) was added and incubated at RT for $30 \mathrm{~min}$. Slides were mounted and optical z series images were obtained using a Zeiss 710 Laser Scanning Confocal Microscope equipped with Gallium Arsenide Phosphide detectors (GAsP) running Zen 2010 Zeiss software. For reconstruction, all serial optical image sections were imported and spatially re-assembled using IMARIS (Bitplane Inc, South Windsor, CT) to generate a 3-dimensional representation of the tissue. All images were also imported into Image Pro Plus (Media Cybernetics Inc.) software for quantitative image analysis.

\section{Quantitative western blotting}

Quantitative Western Blotting was performed with the LiCor immunofluorescence detection system (Licor, Lincoln, NE). Proteins were separated on 4-20\% or 4-12\% SDS Page gels and transferred to nitrocellulose membranes. Primary antibodies were added in $1 / 2 \mathrm{X}$ Odyssey buffer in PBS with $0.1 \%$ Tween 20 . After washing again in TBST, secondary antibodies goat anti-rabbit - IRDye 800 (at 1/5000) and goat anti mouse - IRDye 680 (at $1 / 10,000$ ) for GAPDH, diluted in 1/2X Odyssey buffer in PBS with $0.1 \%$ Tween 20 and $0.01 \%$ SDS were added. Images were acquired on the LiCor Odyssey. In-lane background was removed (Median: Top/Bottom) before analysis with the Odyssey software version 3.0 (LiCor). Protein of interest Integrated intensity values (K counts) were normalized to those of GAPDH.

\section{Mouse strains, treatment and tissue collection}

All animal experiments were performed according to protocols approved by the Institutional Animal Care and Use Committee at The Scripps Research Institute. Mice were housed in a temperature-controlled environment with 12-hr light/dark cycles where they received food and water. GFP-LC3 transgenic mice, which express a GFP-LC3 fusion protein have been described (29) and were obtained from Riken BioResource Center (Koyadai, Japan). Mice were treated for 7 days with $\mathrm{GlcN}(250 \mathrm{mg} / \mathrm{kg})$ administered by gavage or intraperitoneal injection as indicated. Animals were euthanized 8 hours after the last glucosamine administration. For studies on food restriction, 8 weeks old male GFP-LC3 mice were deprived of food for $48 \mathrm{~h}$ with free access to drinking water. Pathogen-free C57BL/6J mice were purchased from the Scripps breeding facility.

\section{Perfusion, fixation, and sectioning of tissues}

A detailed protocol was previously published (30). In brief, mice were perfused with Dulbecco's PBS, and tissues were harvested and fixed using buffered zinc formalin (Z-FIX; Anatech) at RT for $24 \mathrm{~h}$. Then, liver tissue was washed with PBS 1X and stored in PBS 1X at $4^{\circ} \mathrm{C}$. Knee joints were decalcified in TBD-2 (Shandon, Pittsburgh, Pennsylvania USA) for $12 \mathrm{~h}$ on a shaker and then washed and stored in PBS $1 \mathrm{X}$ at $4^{\circ} \mathrm{C}$. Seventy $\mu \mathrm{m}$ sections of liver and knee joints were cut with a Leica VT 1000S vibratome.

\section{Immunostaining of liver and joint vibratome sections}

Free-floating vibratome-cut knee joint and liver sections were permeabilized with $0.5 \%$ Triton X-100 for $2 \mathrm{~h}$ at RT. The sections were incubated with Rhodamine Phalloidin (1:50; Invitrogen) in PBS with $1 \%$ NGS and $0.3 \%$ Triton X-100 at $4{ }^{\circ} \mathrm{C}$ overnight to label F-actin. The sections were washed 5X in PBS and then counterstained with Hoechst 33342 (1:1,000; Invitrogen) for $1 \mathrm{hr}$ at RT to label nuclei, and mounted with ProLong® Gold antifade reagent (Invitrogen) for confocal microscopy.

For immunostaining with LC3-far red, joint vibratome sections were incubated with antiLC3 (MBL, 1:5000) for $1 \mathrm{~h}$ at RT (100 rpm) and then at $4^{\circ} \mathrm{C}$ for 2 days. The sections were 
incubated with Goat anti rabbit-AlexaFluor 568 (1:400) in PBS/0.3\% Triton/1\% NGS for $1 \mathrm{~h}$ at RT (100 rpm). Then, the sections were washed and incubated with Phalloidin/647 in PBS/ $0.3 \%$ triton $/ 1 \% \mathrm{NGS}$ overnight at $4{ }^{\circ} \mathrm{C}$ to label F-actin. After incubation, the sections were washed 5X in PBS and counterstained with Hoechst 33342 (1:1,000; Invitrogen, 998468) for $1 \mathrm{~h}$ at RT to label nuclei, and mounted with ProLong® Gold antifade reagent (Invitrogen) for confocal microscopy.

\section{Quantitative imaging of autophagosome formation in GFP LC3 mice}

A detailed protocol has been published (30). Autophagosomes can be detected as discrete regions of high fluorescence and it allows quantification of not only the number of vesicles per cell, but also their area, perimeter, feret and circularity. Optical z series images were obtained using a Zeiss 710 Laser Scanning Confocal Microscope equipped with Gallium Arsenide Phosphide detectors (GAsP) running Zen 2010 Zeiss software. For reconstruction, all serial optical image sections were imported and spatially re-assembled using IMARIS (Bitplane Inc, South Windsor CT) to generate a 3-dimensional representation of the tissue. All images were also imported into Image Pro Plus (Media Cybernetics Inc.) software for quantitative image analysis.

\section{Statistical analysis}

To evaluate normal distribution of the data, we performed the Kolmogorov-Smirnov test. Statistically significant differences between two groups were determined with unpaired Student's t-test. Statistically significant differences between multiple comparisons were determined by ANOVA with Tukey's multiple comparisons test. P values less than 0.05 were considered significant.

\section{RESULTS}

\section{Glucosamine activates autophagy in normal human chondrocytes}

To determine whether GlcN promotes autophagy activation in chondrocytes, western blot analysis was performed to detect LC3-I lipidation and conversion to LC3-II as a marker of autophagy activation and autophagosome formation (31).

The results indicate a dose-dependent increase in the LC3-II/LC3-I ratio, which was significant at 5 and $10 \mathrm{mM}$ GlcN (Figure 1A). The increase in the LC3-II/LC3-I ratio was already significant at $8 \mathrm{~h}$ (Figure $1 \mathrm{~B})$.

Fluorescence-based detection of LC3 in cultured cells reveals either diffuse cytoplasmic protein or puncta formation, which reflects protein associated with autophagosomes and the number of punctate LC3 is associated with autophagosome number (31). In response to GlcN treatment, we observed an increase in LC3 puncta at $8 \mathrm{~h}(40.91 \pm 3.027$ vs $9.818 \pm$ $1.957, P<0.0001)$ and $24 \mathrm{~h}(46.87 \pm 2.392$ vs $11.82 \pm 2.318, P<0.0001)$. Confocal microscopy showed that the number of LC3-positive vesicles was significantly higher in the GlcN treated cells at 8 and $24 \mathrm{~h}$ (Figure 1C).

\section{GIcN increases autophagy flux in human articular chondrocytes}

Increased autophagosome formation as detected by LC3 lipidation on western blot, or fluorescent LC3 puncta, can reflect either increased autophagic activity, or reduced turnover of autophagosomes. Autophagy flux assays distinguish between the induction of autophagy and inhibition of subsequent events and measure LC3 turnover under conditions where autophagosome-lysosome fusion, lysosomal acidification or lysosomal proteases are inhibited. To examine the autophagy flux, LC3 turnover assays were performed, where chondrocytes were treated with GlcN $10 \mathrm{mM}$ in the presence of bafilomycin $\mathrm{A} 1$, an $\mathrm{H}^{+}-$ 
ATPase inhibitor, or chloroquine, an inhibitor of lysosomal acidification for 8 or 24 hours. The inhibitors increased the LC3-II/LC3-I ratio in the absence of GlcN, suggesting basal activation of autophagy in cultured chondrocytes. When combined with GlcN, bafilomycin $(P<0.05)$ and chloroquine $(P<0.01)$ the LC3II/LC3I ratio increased beyond the level observed with GlcN alone (Figure 2). Both inhibitors showed significant increases at 8 hours. The magnitude of their effect was less at 24 hours but chloroquine still led to a significant difference. The reduced inhibitor effect at 24 hours may be due to the fact that the effect of GlcN is also lower at 24 hours than at 8 hours. These results indicate that GlcN enhances autophagy flux in normal human chondrocytes.

\section{GIcN enhances autophagy by inhibition of Akt/FoxO and mTOR pathway}

To investigate the molecular components of the effect of GlcN on autophagy, we studied Akt, FoxO and mTOR in human chondrocytes. The results indicate that GlcN treatment inhibits phosphorylation of Akt, FoxO3 and rbS6, a direct and downstream target of mTOR (Figure 3A). This inhibition was significant $(\mathrm{P}<0.05$ ) vs control condition (Figure 3B). Phosphorylation of Akt and rbS6 was reduced at 8 hours and decreased further by 24 hours (Figure 3C). These results suggest that GlcN enhancement of autophagy is associated with inhibition of Akt, FoxO3 and mTOR.

\section{Identification and quantification of autophagosomes in the liver of GIcN treated and food restricted mice}

To determine whether GlcN activates autophagy in vivo, we used transgenic reporter mice expressing GFP fused to LC3. In these mice GFP-LC3 is ubiquitously expressed, and the accumulation of GFP puncta indicates the formation of autophagosomes (31). We first analyzed the liver, where autophagy is induced by food restriction. Following 48 hours of food restriction, individual autophagosomes were detected as discrete regions of high fluorescence compared to non-starvation condition (data not shown). More importantly, GlcN administration ( $250 \mathrm{~kg} /$ body weight/day) for 7 days by intraperitoneal injection increased autophagy in liver and the magnitude of autophagy activation was greater in response to GlcN as compared to starvation (Figure 4A). This increase in GFP-LC3 signal in response to GlcN and starvation was significant based on total number of vesicles per cell $(P$ $<0.05)$ and total area of vesicles per cell $\left(\mu \mathrm{m}^{2}\right)(P<0.5)$ (Figure 4B).

\section{Identification and quantification of autophagosomes in the knee joints of GICN treated and food restricted mice}

In response to GlcN treatment by intraperitoneal injection or food restriction, we observed an increase in the GFP-LC3 signal of cartilage from the mouse knee joints (Figure 5). GlcN effects were statistically significant based on the total number of vesicles per cell $(P<0,01)$ and on the total area of vesicles per cell $\left(\mu \mathrm{m}^{2}\right)(P<0.01)$ (Figure 5A). The effects of GlcN on autophagy were also tested upon oral administration. Based on the total number of vesicles per cell and on the total area of vesicles per cell $\left(\mu \mathrm{m}^{2}\right)$ respectively, $(21.37 \pm 4.714$ vs. $7.258 \pm 1.406, P<0.01 ; 8,887 \pm 3.596$ vs. $1.896 \pm 0.4798, P<0.01$ ), both routes of administration led to similar increases in autophagy.

In addition, starvation for $48 \mathrm{~h}$ significantly increased the GFP-LC3 signal in articular cartilage as measured by the total number of vesicles per cell $(P<0,05)$ and total area of vesicles per cell $\left(\mu \mathrm{m}^{2}\right)(P<0.05)$ (Figure 5B). 


\section{Whole reconstruction of tibial plateau showing distribution of GFP-LC3 signal in response to GIcN}

To understand how autophagy is activated in cartilage, we evaluated the levels of GFP-LC3 in response to GlcN on the entire tibial plateau. Serial images using confocal microscopy were taken to reconstruct the tibial plateau in the mouse knee joints. The results indicate that the highest levels of GFP-LC3 signal were observed from the chondrocytes present in the superficial and upper middle zone of the articular cartilage. In contrast, only few cells in the deep zone contained detectable levels of GFP-LC3 signal (Figure 6). These results indicate that chondrocytes from the superficial and upper middle zone are more responsive to upregulation of autophagy.

\section{DISCUSSION}

The present results indicate that GlcN activates autophagy in vitro and in vivo. Autophagy is an essential cellular homeostasis mechanism, which not only functions in adjusting to variations in nutrient supply but importantly in removing dysfunctional organelles and macromolecules (11). A specific form of autophagy, termed mitophagy is responsible for the removal of damaged mitochondria (32), which have been observed in OA-affected cartilage (33). The role of autophagy in cellular and organismal health is illustrated by findings from experimental and spontaneous aging-associated defects in this process. Major manifestations are cell dysfunction, which ranges from abnormal secretory profiles, which include inflammatory mediators and similar to the senescence-associated secretory phenotype to cell death (14). These cellular dysfunctions lead to tissue and organ failure and manifest typically as aging-associated degenerative diseases. Conversely, pharmacological augmentation of autophagy protects against cell dysfunction and disease and in model organisms extends life and health span.

Aging represents the major risk factor for $\mathrm{OA}$ and a spectrum of aging-related changes have been observed in cartilage cells and extracellular matrix (34). Our recent observations show that joint aging in humans and experimental models is associated with autophagy defects in articular cartilage (15). The temporal and mechanistic relationship between this and the previously documented aging associated cellular changes requires further study but available data support the hypothesis that primary autophagy defects can potentially lead to many of the observed aging-associated phenotypes in cartilage.

Rapamycin has been an important tool in autophagy research. It inhibits mTORC1 and thus can lead to autophagy activation. In an animal model of OA induced by joint destabilization, it reduced the severity of joint pathology (17). This study established proof-of concept that autophagy activation has protective effects against cartilage degradation. The long-term use of rapamycin is associated with a substantial risk for adverse events, in part related to its potent immunosuppressive activity (35). Collectively, these observations motivated the present study to determine whether GlcN activates autophagy in cartilage cells in vitro and in vivo. One prior study reported that GlcN activated autophagy in HeLa and COS7 cell lines (36). The present observations are the first to show that GlcN activates autophagy in chondrocytes and, importantly, in liver and cartilage. The GlcN effect was observed with similar magnitude upon subcutaneous or oral administration to mice, suggesting that the oral route, which is used clinically, is feasible for autophagy activation. Starvation, a well-known activator of autophagy also increased autophagosome formation in cartilage. Potential mechanism by which GlcN activates autophagy in vivo include direct effects on cells in liver or cartilage. Alternatively, it is possible that indirect effects are involved. For example GlcN may induce in liver the production of secreted factors that induce autophagy in cartilage and other tissues. 
Using the GFP-LC3 autophagy reporter mouse model we provide the first in vivo analysis of basal autophagy in cartilage. There is a specific pattern with highest levels in the cartilage superficial zone and in the weight bearing regions. More interestingly, the increased autophagy activation in response to GlcN is most profound in the superficial and upper middle zone, the same areas where autophagy regulators are decreased with aging in human and murine cartilage. The superficial zone is also where the earliest structural defects occur in OA.

GlcN has been shown to regulate various chondrocyte functions in vitro (37). The relationship between the present finding of autophagy activation and the prior observations requires further investigation. GlcN also showed therapeutic efficacy in animal models of OA, including in the spontaneous models in Str mice (25) and guinea pigs (38). The present observations suggest the hypothesis that these in vivo effects may be in part mediated by a GlcN effect on autophagy.

In the present study, in vitro effects of GlcN were observed between 1 to $10 \mathrm{mM}$ and for the in vivo studies mice were treated with $250 \mathrm{mg} / \mathrm{kg} / \mathrm{day}$. These dosages are consistent with many prior in vitro $(26-28,37)$ and animal model studies $(25,38)$. Local tissue concentrations similar to the in vitro concentrations used in this study can probably not be reached following ingestion of the typical $1500 \mathrm{mg}$ dose in humans (39). The GlcN concentrations used in the animal model studies were also much higher. Lower concentrations were not tested in but may be effective. Alternatively, GlcN analogs or formulations may be able reach local concentrations in the joint tissues that are sufficient to activate autophagy.

mTOR is considered as a main regulator of autophagy, and the mTOR inhibitor rapamycin induces autophagy in many cell types $(40,41)$ including articular chondrocytes in which prevents cartilage damage (17). GlcN treatment has shown efficacy in animal models of OA (23-25), potentially through anti-inflammatory and anabolic effects on cartilage cells (26-28). However, additional molecular mechanisms might be involved on GlcN therapeutic effects, including the activation of the autophagy pathway. In this regard, our results show that the GlcN treatment inhibited the mTOR signaling pathway in articular chondrocytes, as reflected by the reduced phosphorylation of ribosomal protein S6, a downstream target of mTOR, which integrates protein synthesis with cell growth and proliferation. To address how mTOR is regulated by GlcN, we examined Akt and FoxO, upstream regulators of mTOR. Our results indicate that GlcN reduces the phosphorylation of Akt and FoxO3 in articular chondrocytes, consistent with the known interaction of Akt and FoxO3 (42). In addition, several reports indicate that FoxO negatively regulates mTOR signaling $(42,43)$ and this is required to activate autophagy. Collectively, our results show that GlcN enhances autophagy in vitro and in vivo and this effect is mainly dependent on the Akt/FoxO/mTOR signaling pathway.

The finding that GlcN activates autophagy raises possibilities that GlcN can be applied to maintain cellular homeostasis and joint health. It may be protective against defective autophagy associated with increased mechanical stress (16) or joint aging (15). Future studies need to determine whether GlcN can alleviate the aging-associated defects in autophagy.

\section{Acknowledgments}

The authors acknowledge Mehrdad Alirezaei for technical assistance.

Funding This study was supported by Cargill, Inc., National Institutes of Health grant AG007996, and the Sam and Rose Stein Endowment Fund. BC was supported by Fondo Investigación Sanitaria-Spain (CD11/00095). 


\section{REFERENCES}

1. Zhang Y, Jordan JM. Epidemiology of osteoarthritis. Clin Geriatr Med. 2010; 26:355-69. [PubMed: 20699159]

2. Loeser RF. Aging and osteoarthritis: the role of chondrocyte senescence and aging changes in the cartilage matrix. Osteoarthritis Cartilage. 2009; 17:971-9. [PubMed: 19303469]

3. Zhang W, Nuki G, Moskowitz RW, Abramson S, Altman RD, Arden NK, et al. OARSI recommendations for the management of hip and knee osteoarthritis: part III: Changes in evidence following systematic cumulative update of research published through January 2009. Osteoarthritis Cartilage. 2010; 18:476-99. [PubMed: 20170770]

4. Lapane KL, Sands MR, Yang S, McAlindon TE, Eaton CB. Use of complementary and alternative medicine among patients with radiographic-confirmed knee osteoarthritis. Osteoarthritis Cartilage. 2012; 20:22-8. [PubMed: 22033041]

5. Henrotin Y, Mobasheri A, Marty M. Is there any scientific evidence for the use of glucosamine in the management of human osteoarthritis? Arthritis Res Ther. 2012; 14:201. [PubMed: 22293240]

6. Goldring MB, Goldring SR. Osteoarthritis. J Cell Physiol. 2007; 213:626-34. [PubMed: 17786965]

7. Wang M, Shen J, Jin H, Im HJ, Sandy J, Chen D. Recent progress in understanding molecular mechanisms of cartilage degeneration during osteoarthritis. Ann N Y Acad Sci. 2011; 1240:61-9. [PubMed: 22172041]

8. Hellio Le Graverand-Gastineau MP. OA clinical trials: current targets and trials for OA. Choosing molecular targets: what have we learned and where we are headed? Osteoarthritis Cartilage. 2009; 17:1393-401. [PubMed: 19426849]

9. Hunter DJ. Pharmacologic therapy for osteoarthritis--the era of disease modification. Nat Rev Rheumatol. 2011; 7:13-22. [PubMed: 21079644]

10. Lotz MK, Carames B. Autophagy and cartilage homeostasis mechanisms in joint health, aging and OA. Nat Rev Rheumatol. 2011; 7:579-87. [PubMed: 21808292]

11. Mizushima N. Physiological functions of autophagy. Curr Top Microbiol Immunol. 2009; 335:7184. [PubMed: 19802560]

12. Zhou H, Huang S. The complexes of mammalian target of rapamycin. Curr Protein Pept Sci. 2010; 11:409-24. [PubMed: 20491627]

13. Fenton TR, Gout IT. Functions and regulation of the 70kDa ribosomal S6 kinases. Int J Biochem Cell Biol. 2011; 43:47-59. [PubMed: 20932932]

14. Rubinsztein DC, Marino G, Kroemer G. Autophagy and aging. Cell. 2011; 146:682-95. [PubMed: 21884931]

15. Carames B, Taniguchi N, Otsuki S, Blanco FJ, Lotz M. Autophagy is a protective mechanism in normal cartilage, and its aging-related loss is linked with cell death and osteoarthritis. Arthritis Rheum. 2010; 62:791-801. [PubMed: 20187128]

16. Carames B, Taniguchi N, Seino D, Blanco FJ, D’Lima D, Lotz M. Mechanical injury suppresses autophagy regulators and its pharmacological activation results in chondroprotection. Arthritis Rheum. 2012; 64:1182-92. [PubMed: 22034068]

17. Carames B, Hasegawa A, Taniguchi N, Miyaki S, Blanco FJ, Lotz M. Autophagy activation by rapamycin reduces severity of experimental osteoarthritis. Ann Rheum Dis. 2012; 71:575-81. [PubMed: 22084394]

18. Klumpen HJ, Beijnen JH, Gurney H, Schellens JH. Inhibitors of mTOR. Oncologist. 2010; 15:1262-9. [PubMed: 21147869]

19. Anderson JW, Nicolosi RJ, Borzelleca JF. Glucosamine effects in humans: a review of effects on glucose metabolism, side effects, safety considerations and efficacy. Food Chem Toxicol. 2005; 43:187-201. [PubMed: 15621331]

20. Towheed TE, Maxwell L, Anastassiades TP, Shea B, Houpt J, Robinson V, et al. Glucosamine therapy for treating osteoarthritis. Cochrane Database Syst Rev. 2005:CD002946. [PubMed: 15846645]

21. Simon RR, Marks V, Leeds AR, Anderson JW. A comprehensive review of oral glucosamine use and effects on glucose metabolism in normal and diabetic individuals. Diabetes Metab Res Rev. 2011; 27:14-27. [PubMed: 21218504] 
22. Altman RD. Glucosamine therapy for knee osteoarthritis: pharmacokinetic considerations. Expert Rev Clin Pharmacol. 2009; 2:359-71. [PubMed: 22112180]

23. Tiraloche G, Girard C, Chouinard L, Sampalis J, Moquin L, Ionescu M, et al. Effect of oral glucosamine on cartilage degradation in a rabbit model of osteoarthritis. Arthritis Rheum. 2005; 52:1118-28. [PubMed: 15818693]

24. Wen ZH, Tang CC, Chang YC, Huang SY, Hsieh SP, Lee CH, et al. Glucosamine sulfate reduces experimental osteoarthritis and nociception in rats: association with changes of mitogen-activated protein kinase in chondrocytes. Osteoarthritis Cartilage. 2010; 18:1192-202. [PubMed: 20510383]

25. Chiusaroli R, Piepoli T, Zanelli T, Ballanti P, Lanza M, Rovati LC, et al. Experimental pharmacology of glucosamine sulfate. Int J Rheumatol. 2011; 2011:939265. [PubMed: 22007227]

26. Gouze JN, Gouze E, Popp MP, Bush ML, Dacanay EA, Kay JD, et al. Exogenous glucosamine globally protects chondrocytes from the arthritogenic effects of IL-1beta. Arthritis Res Ther. 2006; 8:R173. [PubMed: 17109745]

27. Imagawa K, de Andres MC, Hashimoto K, Pitt D, Itoi E, Goldring MB, et al. The epigenetic effect of glucosamine and a nuclear factor-kappa B (NF-kB) inhibitor on primary human chondrocytes-implications for osteoarthritis. Biochem Biophys Res Commun. 2011; 405:362-7. [PubMed: 21219853]

28. Largo R, Alvarez-Soria MA, Diez-Ortego I, Calvo E, Sanchez-Pernaute O, Egido J, et al. Glucosamine inhibits IL-1beta-induced NFkappaB activation in human osteoarthritic chondrocytes. Osteoarthritis Cartilage. 2003; 11:290-8. [PubMed: 12681956]

29. Mizushima N, Yamamoto A, Matsui M, Yoshimori T, Ohsumi Y. In vivo analysis of autophagy in response to nutrient starvation using transgenic mice expressing a fluorescent autophagosome marker. Mol Biol Cell. 2004; 15:1101-11. [PubMed: 14699058]

30. Alirezaei M, Kemball CC, Flynn CT, Wood MR, Whitton JL, Kiosses WB. Short-term fasting induces profound neuronal autophagy. Autophagy. 2010; 6:702-10. [PubMed: 20534972]

31. Mizushima N, Yoshimori T, Levine B. Methods in mammalian autophagy research. Cell. 2010; 140:313-26. [PubMed: 20144757]

32. Green DR, Galluzzi L, Kroemer G. Mitochondria and the autophagy-inflammation-cell death axis in organismal aging. Science. 2011; 333:1109-12. [PubMed: 21868666]

33. Blanco FJ, Rego I, Ruiz-Romero C. The role of mitochondria in osteoarthritis. Nat Rev Rheumatol. 2011; 7:161-9. [PubMed: 21200395]

34. Lotz M, Loeser RF. Effects of aging on articular cartilage homeostasis. Bone. 2012

35. Sofroniadou S, Goldsmith D. Mammalian target of rapamycin (mTOR) inhibitors: potential uses and a review of haematological adverse effects. Drug Saf. 2011; 34:97-115. [PubMed: 21247219]

36. Shintani T, Yamazaki F, Katoh T, Umekawa M, Matahira Y, Hori S, et al. Glucosamine induces autophagy via an mTOR-independent pathway. Biochem Biophys Res Commun. 2010; 391:17759. [PubMed: 20045674]

37. Block JA, Oegema TR, Sandy JD, Plaas A. The effects of oral glucosamine on joint health: is a change in research approach needed? Osteoarthritis Cartilage. 2010; 18:5-11. [PubMed: 19733270]

38. Taniguchi S, Ryu J, Seki M, Sumino T, Tokuhashi Y, Esumi M. Long-term oral administration of glucosamine or chondroitin sulfate reduces destruction of cartilage and up-regulation of MMP-3 mRNA in a model of spontaneous osteoarthritis in Hartley guinea pigs. J Orthop Res. 2012; 30:673-8. [PubMed: 22058013]

39. Jackson CG, Plaas AH, Sandy JD, Hua C, Kim-Rolands S, Barnhill JG, et al. The human pharmacokinetics of oral ingestion of glucosamine and chondroitin sulfate taken separately or in combination. Osteoarthritis Cartilage. 2010; 18:297-302. [PubMed: 19912983]

40. Shigemitsu K, Tsujishita Y, Hara K, Nanahoshi M, Avruch J, Yonezawa K. Regulation of translational effectors by amino acid and mammalian target of rapamycin signaling pathways. Possible involvement of autophagy in cultured hepatoma cells. J Biol Chem. 1999; 274:1058-65. [PubMed: 9873051]

41. Sabers CJ, Martin MM, Brunn GJ, Williams JM, Dumont FJ, Wiederrecht G, et al. Isolation of a protein target of the FKBP12-rapamycin complex in mammalian cells. J Biol Chem. 1995; 270:815-22. [PubMed: 7822316] 
42. Mammucari C, Milan G, Romanello V, Masiero E, Rudolf R, Del Piccolo P, et al. FoxO3 controls autophagy in skeletal muscle in vivo. Cell Metab. 2007; 6:458-71. [PubMed: 18054315]

43. Jia K, Chen D, Riddle DL. The TOR pathway interacts with the insulin signaling pathway to regulate C. elegans larval development, metabolism and life span. Development. 2004; 131:3897906. [PubMed: 15253933] 

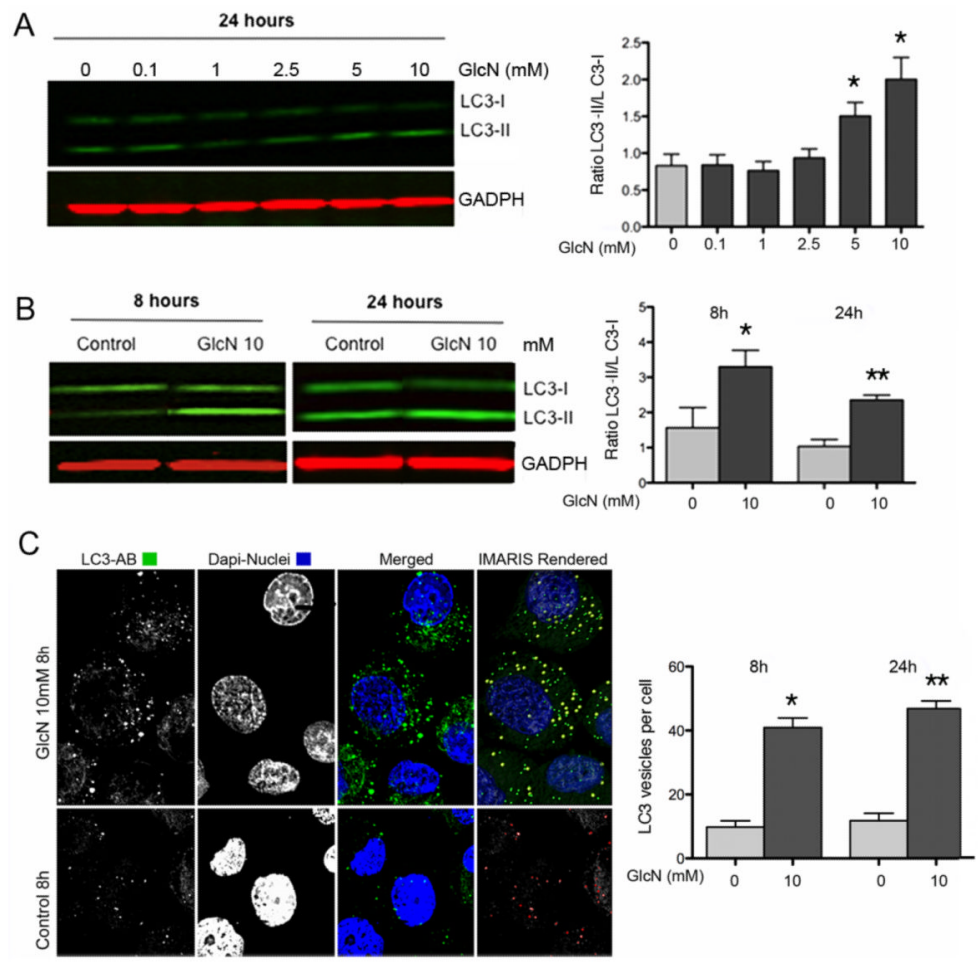

Figure 1. GlcN and autophagy activation in human articular chondrocytes

A, Normal human chondrocytes were untreated (DMEM $2 \%$ CS) or treated with GlcN $(0.1$, $1,2.5,5$ and $10 \mathrm{mM}$ ) for $24 \mathrm{~h}$. Total protein was analyzed by western blotting using antiLC3 and GAPDH. Values represent mean \pm SD of three separate experiments, $*=\mathrm{P}<0.01$ vs. control. Bar graphs show quantification of western signals expressed as LC3II/LC3I ratios. B, Normal human chondrocytes were untreated (DMEM 2\% CS) or treated with GlcN (10 mM) for 8 or $24 \mathrm{~h}$. LC3 was analyzed by western blotting. C, Normal human chondrocytes were untreated (DMEM 2\% CS) or treated with GlcN (10 mM) for 8 and 24 hours stained with anti-LC3 and analyzed by confocal microscopy. The right-upper panel series represents a 3D IMARIS rendered reconstruction of the left-panels. The right-bottom panel represents vesicles that were outlined by Image Pro software (outlined in red) for quantification of vesicles. Magnification: 63x. The bar graph shows quantification of LC3positive vesicles at 8 and 24 hours. 
A

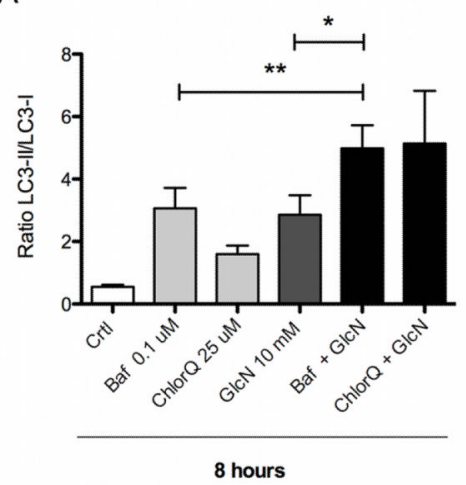

B

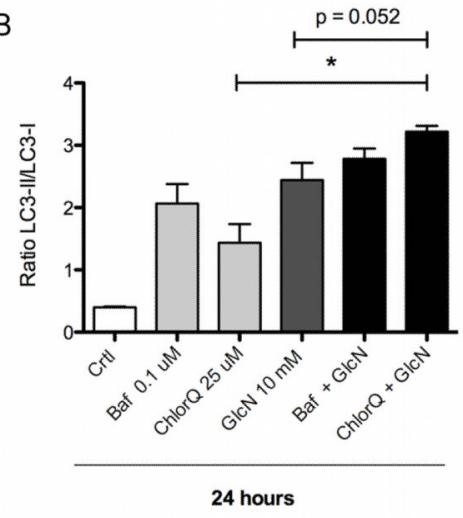

Figure 2. GlcN increases autophagy flux in human articular chondrocytes A, Normal human chondrocytes were untreated (DMEM 2\% CS) or treated with $10 \mathrm{mM}$ GlcN for $8 \mathrm{~h}$ or $24 \mathrm{~h}$, with or without Bafilomycin (Baf; $0.1 \mu \mathrm{M}$ ) or Chloroquine (ChlorQ; 25 $\mu \mathrm{M})$. Protein extracts were analyzed by western blotting using anti-LC3 and GAPDH. B, Bar graphs show quantification of western signals expressed as LC3II/LC3I ratios. Values represent mean $\pm \mathrm{SD}$ of three separate experiments, $*=\mathrm{P}<0.001$ vs. control; $* *=\mathrm{P}<0.05$ vs. GlcN; $\&=\mathrm{P}<0.01$ vs. GlcN. 


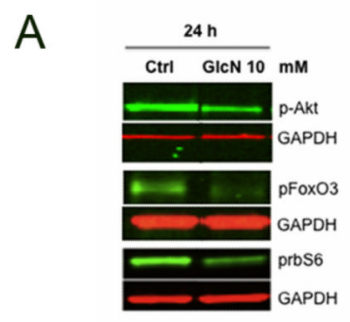

B

\begin{tabular}{|c|c|c|}
\hline Protein of interest & Control & GIcN 10 mM \\
\hline p-Akt & $0.263 \pm 0.046$ & $0.098 \pm 0.014^{\circ}$ \\
\hline pFoxO3 & $0.066 \pm 0.019$ & $0.010 \pm 0.002^{\circ}$ \\
\hline prbS6 & $1.029 \pm 0.180$ & $0.277 \pm 0.060^{\circ}$ \\
\hline
\end{tabular}

C

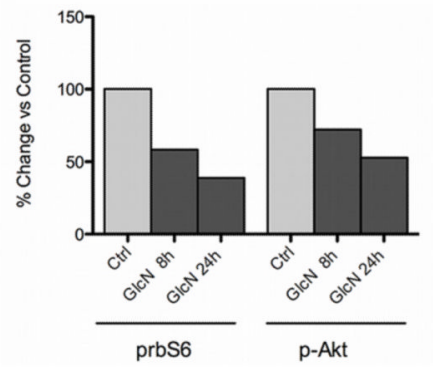

Figure 3. Glucosamine modulates Akt/mTOR pathway in human chondrocytes A. Normal human chondrocytes were untreated (DMEM 2\% CS) or treated with GlcN (10 $\mathrm{mM}$ ) for $24 \mathrm{~h}$. Protein extracts were analyzed by western blotting using antibodies to p-Akt, pFOXO3, prbS6 and GAPDH. B, Bar graphs show quantification of western signals of pAkt, pFoxO3 and prbS6 normalized by GAPDH. Values represent mean \pm SD of three separate experiments for $\mathrm{p}$-Akt and $\mathrm{pFoxO} 3, *=\mathrm{P}<0.05$ vs. control $24 \mathrm{~h}$ and values represent mean \pm SD of 9 separate experiments for prbS6, $* *=\mathrm{P}<0.01$ vs. control $24 \mathrm{~h}$. C, Comparison of p-Akt and prbS6 at $8 \mathrm{~h}$ and $24 \mathrm{~h}$ in chondrocytes treated with $\mathrm{GlcN}(10 \mathrm{mM})$. 


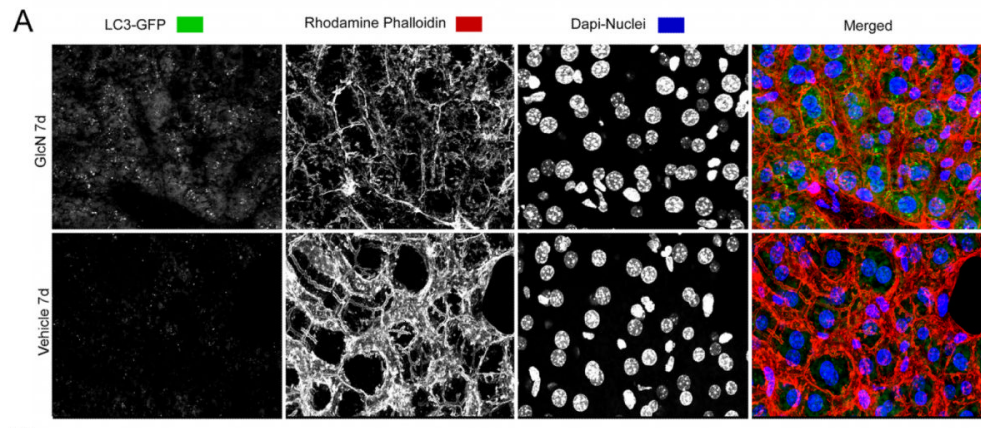

B

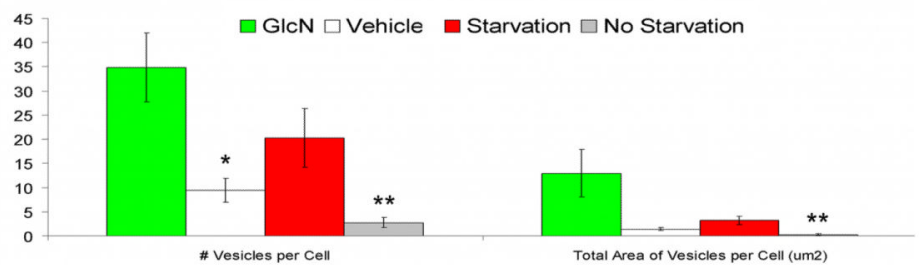

Figure 4. Identification and quantification of autophagosomes in the livers of GlcN treated and food restricted mice

GFP-LC3 transgenic mice were treated with vehicle or GlcN (250 mg/kg body weight/day) for 7 days by i.p. injection or subjected to food restriction for $48 \mathrm{~h}$ to activate autophagy. Induction of autophagy was analyzed in vibratome-cut sections by confocal microscopy. A, Representative images of GFP-LC3 were stained with Rhodamine Phalloidin and Hoechst 33342 to label F-actin and nuclei, respectively. Two sets of control mice were included: vehicle treated or normal-fed GFP-LC3 mice, to provided a baseline for autophagic activity in the liver and a wild-type C57BL/6 mice to determine the background level of green fluorescence. B, Quantitative analysis of vesicles (autophagosomes) in hepatocytes, including the total number of vesicles per cell and the total area of vesicles per cell $\left(\mu \mathrm{m}^{2}\right)$. GlcN treatment: $*=\mathrm{P}<0.05$ vs. vehicle; Starvation: $* *=\mathrm{P}<0.05$ vs. non starvation. Vehicle indicates standard diet without GlcN. Values represent average \pm SEM of four animals per group. Magnification: 63x. 


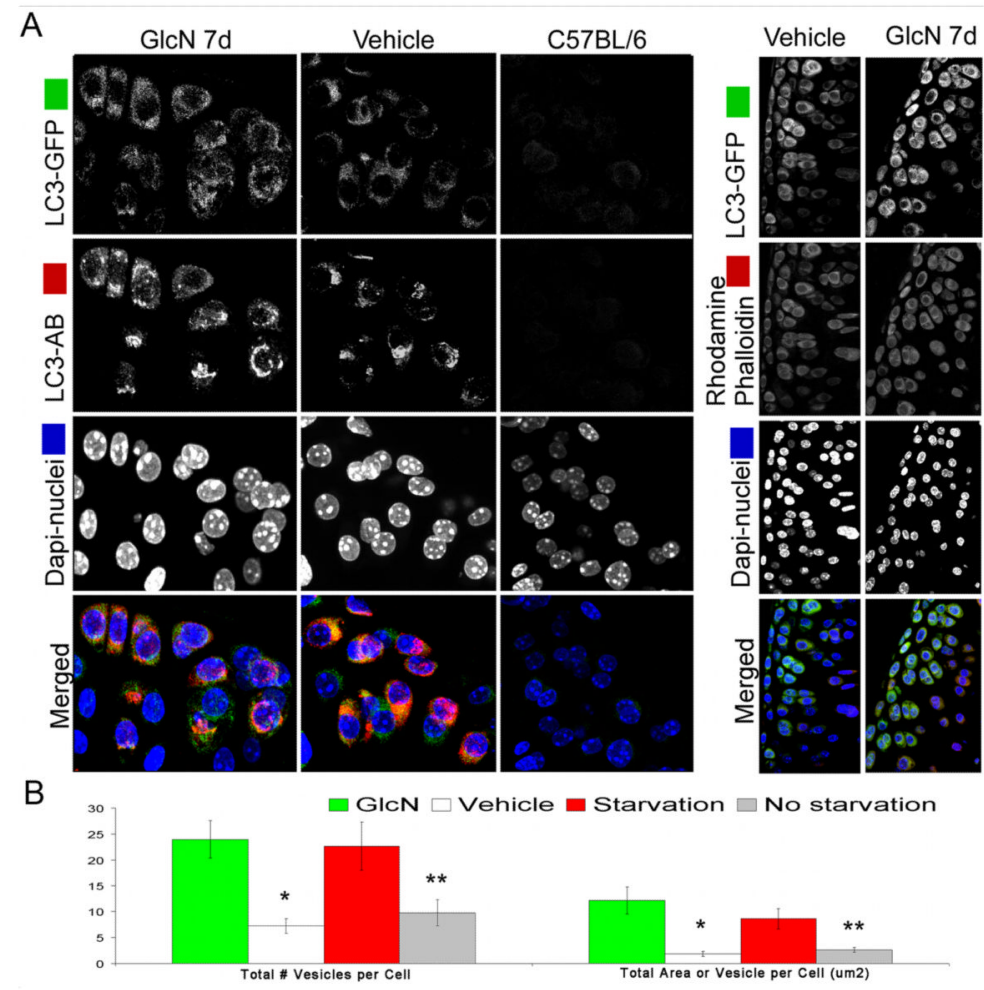

Figure 5. Identification and quantification of autophagosomes in the joints of GlcN treated and food restricted mice

GFP-LC3 transgenic mice were treated as described in Figure 4. Mice were perfused with PBS, and the knee joints were harvested and sagittal vibratome-cut sections $(70 \mu \mathrm{m})$ were stained with Rhodamine Phalloidin, anti-LC3 far red and Hoechst 33342 to label F-actin, LC3 and nuclei, respectively, and then analyzed by confocal microscopy. A, Representative images of GFP-LC3 signal in response to GlcN treatment are shown. A merged fluorescence image is shown in the bottom panel; GFP-LC3 (green), LC3 far red (red), F-actin (red), nuclei (blue). Two sets of control mice were included as described in Figure 4. B, Quantitative analysis of vesicles (autophagosomes) in chondrocytes, including the total number of vesicles per cell and the total area of vesicles per cell $\left(\mu \mathrm{m}^{2}\right)$. GlcN treatment: * = $\mathrm{P}<0.01$ vs. vehicle; Starvation: $* *=\mathrm{P}<0.05$ vs. non starvation. Vehicle indicates standard diet without GlcN. Values represent average \pm SEM of four animals per group. Magnification: 63x. 

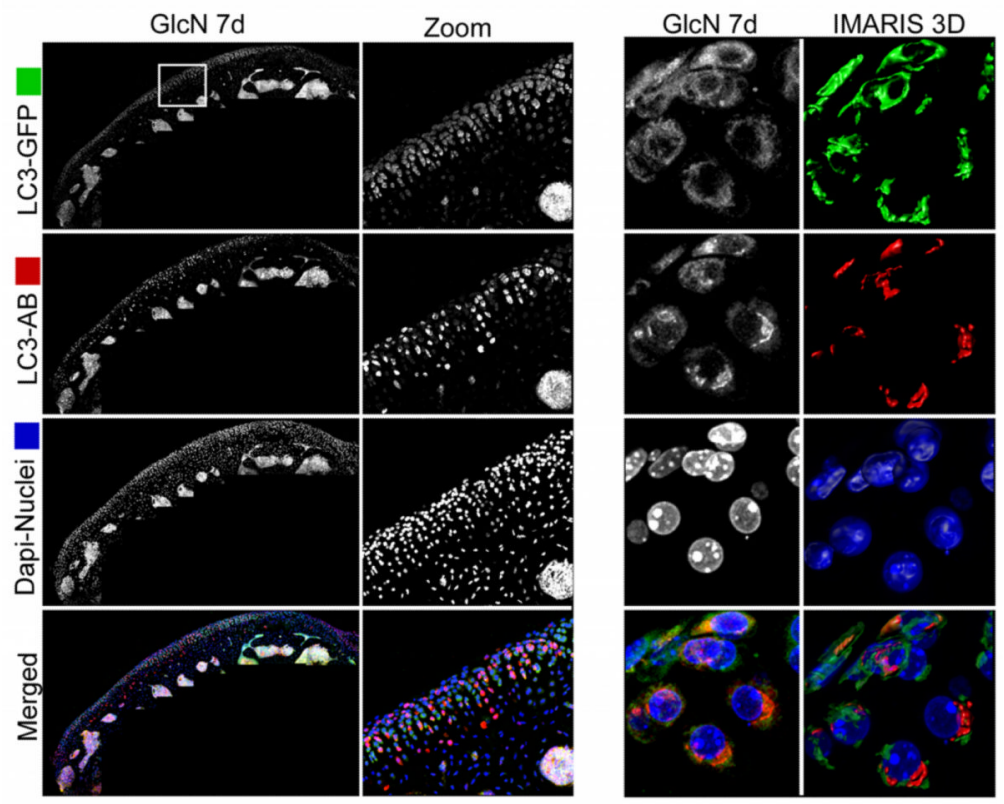

Figure 6. Whole reconstruction of tibial plateau showing distribution of GFP-LC3 signal in response to GlcN

GFP-LC3 transgenic mice were treated with GlcN and knee joints were collected as described in Figure 5. Sagittal vibratome-cut sections were stained with anti-LC3 far red and Hoechst 33342 to label LC3 and the nuclei, respectively and then analyzed by confocal microscopy. Representative images of GFP-LC3 signal in response to GlcN treatment from cartilage knee joints are shown. A merged fluorescence image is shown in the bottom panel; GFP-LC3 (green), LC3 (red), nuclei (blue). The 4th panel (right) series represents a 3D IMARIS rendered reconstruction of the 3rd panel series, where maximum induction is observed. 